\title{
Critical Temperature of Interacting Bose Gases in Periodic Potentials
}

\author{
T. T. Nguyen, ${ }^{1,2}$ A. J. Herrmann, ${ }^{3}$ M. Troyer, ${ }^{4}$ and S. Pilati ${ }^{1}$ \\ ${ }^{1}$ The Abdus Salam International Centre for Theoretical Physics, 34151 Trieste, Italy \\ ${ }^{2}$ SISSA-International School for Advanced Studies, 34136 Trieste, Italy \\ ${ }^{3}$ Department of Physics, University of Fribourg, 1700 Fribourg, Switzerland \\ ${ }^{4}$ Theoretische Physik, ETH Zurich, 8093 Zurich, Switzerland
}

(Received 4 December 2013; revised manuscript received 16 February 2014; published 29 April 2014)

\begin{abstract}
The superfluid transition of a repulsive Bose gas in the presence of a sinusoidal potential which represents a simple-cubic optical lattice is investigated using quantum Monte Carlo simulations. At the average filling of one particle per well the critical temperature has a nonmonotonic dependence on the interaction strength, with an initial sharp increase and a rapid suppression at strong interactions in the vicinity of the Mott transition. In an optical lattice the positive shift of the transition is strongly enhanced compared to the homogenous gas. By varying the lattice filling we find a crossover from a regime where the optical lattice has the dominant effect to a regime where interactions dominate and the presence of the lattice potential becomes almost irrelevant.
\end{abstract}

DOI: 10.1103/PhysRevLett.112.170402

PACS numbers: 05.30.Jp, 03.75.Hh, 67.10.-j

The combined effect of interparticle interactions and external potentials plays a fundamental role in determining the quantum-coherence properties of several many-body systems, including $\mathrm{He}$ in Vycor or on substrates, paired electrons in superconductors and in Josephson junction arrays, neutrons in the crust of neutron stars [1], and ultracold atoms in optical potentials. However, even the (apparently) simple problem of calculating the superfluid transition temperature $T_{c}$ of a dilute homogeneous Bose gas has challenged theoreticians for decades [2]. Many techniques have been employed, obtaining contradicting results, differing even in the functional dependence of $T_{c}$ on the interaction parameter (the two-body scattering length $a$ ) and in the sign of the shift with respect to the ideal gas transition temperature $T_{c}^{0}$ (for a review see Ref. [3]). In the weakly interacting limit, the shift of the critical temperature $\Delta T_{c}=T_{c}-T_{c}^{0}$ due to interactions has a linear dependence $\Delta T_{c} / T_{c}^{0} \simeq c n^{1 / 3} a[4,5]$, where $n$ is the density and the coefficient $c=1.29(5)$ was determined using Monte Carlo simulations of a classical-field model defined on a discrete lattice [6,7]. Continuous-space quantum Monte Carlo simulations of Bose gases with short-range repulsive interactions have shown that this linear form is valid only in the regime $n^{1 / 3} a \lesssim 0.01$, while at stronger interaction $T_{c}$ reaches a maximum where $\Delta T_{c} / T_{c}^{0} \simeq 6.5 \%$ and then decreases for $n^{1 / 3} a \gtrsim 0.2$ [8]. This suppression of $T_{c}$ occurs in a regime where universality in terms of the scattering length is lost and other details of the interaction potential become relevant [8-10]. In recent years ultracold atomic gases have emerged as the ideal experimental test bed for many-body theories [11]. However, the direct measurement of interactions effects on $T_{c}$ has been hindered by the presence of the harmonic trap. In the presence of confinement the main interactions effect can be predicted by mean-field theory and is due to the broadening of the density profile [12], leading to a suppression of $T_{c}$. Deviations from the mean-field prediction and effects due to critical correlations have been measured in Refs. [13,14]. A major breakthrough has been achieved recently with the realization of Bose-Einstein condensation in quasiuniform trapping potentials [15]. This setup allows a more direct investigation of critical points where a correlation length diverges and the arguments based on the local density approximation become invalid. Alternatively, critical properties can be extracted directly from experiments with harmonic confinements by using trap-size scaling at fixed chemical potential [16].

The superfluid transition in the presence of periodic potentials is even more complex than in homogeneous systems due to the intricate interplay between interparticle interactions and the external potential and to the role of commensurability. In this Letter, we employ unbiased quantum Monte Carlo methods to determine the critical temperature of a 3D repulsive Bose gas in the presence of a simple-cubic optical lattice with spacing $d$. We find that at the integer filling $n d^{3}=1$ (an average density of one bosons per well of the external field) the critical temperature $T_{c}$ has an intriguing nonmonotonic dependence on the interaction strength (parametrized by the ratio $a / d$ ) with an initial sharp increase in the regime of small $a / d$ followed by a rapid suppression terminating at the Mott insulator quantum phase transition. While the simulations of approximate discrete lattice models indicate that repulsive interactions suppress superfluidity [17], we find instead that repulsion and spatial inhomogeneity due to a moderately intense periodic potential can cooperate to give an increase of $T_{c}$ which is even more pronounced than in homogenous systems (see Fig. 1). By varying the filling 


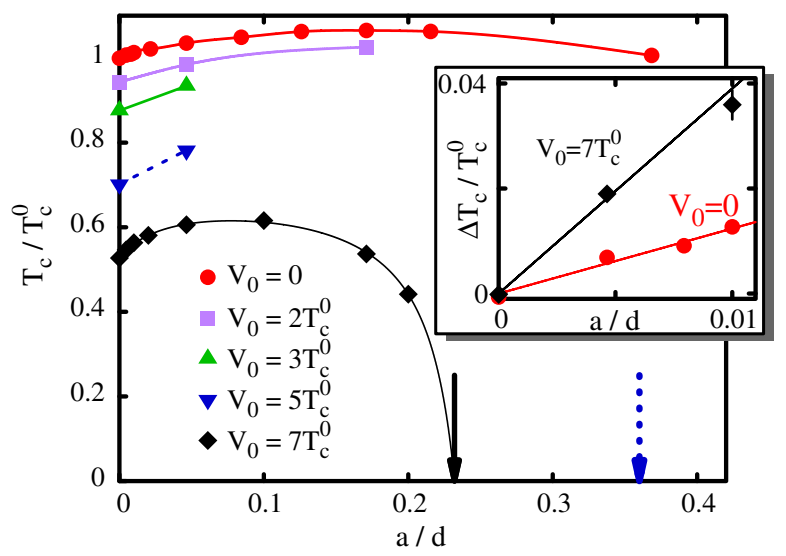

FIG. 1 (color online). Main panel: Superfluid transition temperature $T_{c} / T_{c}^{0}$ as a function of the scattering length $a$, for different intensities of the optical lattice $V_{0}$. The filling is fixed at $n d^{3}=1$ ( $n$ is the density and $d$ the lattice spacing). The vertical arrows indicate the $T=0$ Mott insulator transition for $V_{0}=7 T_{c}^{0}$ (black) and $V_{0}=5 T_{c}^{0}$ (dashed blue lines) [35]. The lines are guides to the eye. Inset: Shift of $T_{c}$ with respect to the value at $a=0$. The solid lines are linear fits. The transition temperature of the homogeneous noninteracting gas $\left(V_{0}=0\right.$ and $\left.a=0\right)$ is $T_{c}^{0} \cong 0.671 E_{R}$, where $E_{R}$ is the recoil energy.

$n d^{3}$ at fixed interaction parameter $a / d$, we observe a crossover from a low-density regime where the effect of interactions is marginal and $T_{c}$ is essentially the same as in the noninteracting case, to a regime at large $n d^{3}$ where the role of interactions is dominant while the effect of the optical lattice becomes almost negligible and $T_{c}$ approaches the homogeneous gas value. In the crossover region we observe that $T_{c}$ varies linearly with $n d^{3}$ (see Fig. 2). In our simulations we consider a gas of spinless bosons described by the Hamiltonian

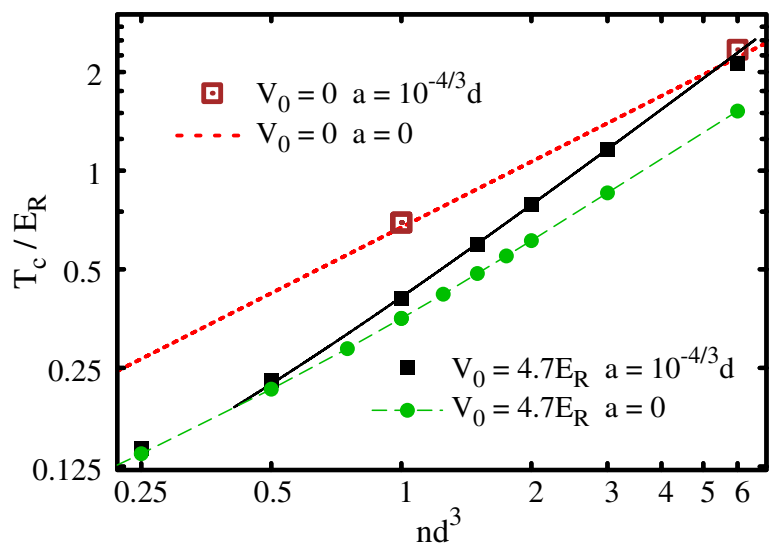

FIG. 2 (color online). Critical temperature $T_{c} / E_{R}$ as a function of the filling factor $n d^{3}$ for fixed interaction strength $a / d$. The red dashed line is the critical temperature of the homogeneous noninteracting Bose gas $T_{c}^{0} \propto n^{2 / 3}$. The black solid line is a linear fit on $T_{c}$ of the interacting gas in the optical lattice in the range $0.5 \leq n d^{3} \leq 3$. The long-dashed green line is a guide to the eye.

$$
H=\sum_{i=1}^{N}\left(-\frac{\hbar^{2}}{2 m} \nabla_{i}^{2}+V\left(\mathbf{r}_{i}\right)\right)+\sum_{i<j} v\left(\left|\mathbf{r}_{i}-\mathbf{r}_{j}\right|\right),
$$

where $\hbar$ is the reduced Planck constant, $m$ the particle mass, and the vectors $\mathbf{r}_{i}$ denote the coordinates of the $N$ particles labeled by the index $i$. The pairwise interparticle interactions are modeled by the hard-sphere potential: $v(r)=+\infty$ if $r<a$ and zero otherwise, where the hardsphere diameter $a$ corresponds to the $s$-wave scattering length. $V(\mathbf{r})=V_{0} \sum_{\alpha=x, y, z} \sin ^{2}(\alpha \pi / d)$ is a simple-cubic optical lattice potential with spacing $d$ and intensity $V_{0}$, which we shall express in units of $T_{c}^{0} \cong 3.3125 \hbar^{2} n^{2 / 3} / m$ or recoil energy $E_{R}=\hbar^{2} \pi^{2} /\left(2 m d^{2}\right.$ ) (we set the Boltzmann constant $\left.k_{B}=1\right)$. The bosons are in a cubic box of volume $V=\left(N_{s} d\right)^{3}$ (where $N_{s}$ is an integer) with periodic boundary conditions.

To simulate the thermodynamic properties of the Hamiltonian (1) we employ a path integral Monte Carlo (PIMC) technique [19] based on the worm algorithm [20]. PIMC provides unbiased estimates of thermal averages of physical quantities using the many-particle configurations $\mathbf{R}=\left(\mathbf{r}_{1}, \ldots, \mathbf{r}_{N}\right)$ sampled from a probability distribution proportional to the density matrix $\rho(\mathbf{R}, \mathbf{R}, T)=$ $\left\langle\mathbf{R}\left|e^{-H / T}\right| \mathbf{R}\right\rangle$ at the temperature $T$ (for more details on our implementation of PIMC, see Refs. [10,20,21] and the Supplemental Material [22]). We are interested in the superfluid fraction $\rho_{S} / \rho$ (where $\rho=m n$ is the total mass density), obtained from the winding number estimator [23], and in the one-body density matrix $n_{1}\left(\mathbf{r}, \mathbf{r}^{\prime}\right)=\left\langle\psi^{\dagger}(\mathbf{r}) \psi\left(\mathbf{r}^{\prime}\right)\right\rangle$, where $\psi^{\dagger}(\mathbf{r})[\psi(\mathbf{r})]$ is the bosonic creation [annihilation] operator.

The critical temperature $T_{c}$ is determined from a finitesize scaling analysis of $\rho_{s} / \rho$ using the scaling ansatz [24],

$$
N^{1 / 3} \rho_{S}(t, N) / \rho=f\left(t N^{1 / 3 \nu}\right)=f(0)+f^{\prime}(0) t N^{1 / 3 \nu}+\cdots .
$$

Here, $t=\left(T-T_{c}\right) / T_{c}$ is the reduced temperature, $\nu$ is the critical exponent of the correlation length $\xi \sim t^{-\nu}$, and $f(x)$ is an analytic function (universal apart from a rescaling of the argument) which allows for a linear expansion close to $x=0$. The scaling ansatz (2) is based on the Josephson relation [25]; we make no assumption on the universality class of the Hamiltonian (1) and obtain $T_{c}, f(0), f^{\prime}(0)$, and $\nu$ from a best fit analysis of PIMC data corresponding to various system sizes [26]. The fitted values of $\nu$ appear to be consistent with the critical exponent of the 3D $X Y$ model $\nu \simeq 0.67$ [28] in the interacting case, and with $\nu=1$ (corresponding to the Gaussian complex field model) in the noninteracting case [29]. In agreement with the scaling ansatz (2), the PIMC results for the rescaled superfluid fraction $N^{1 / 3} \rho_{S} / \rho$ plotted as a function of the rescaled reduced temperature $N^{1 / 3 v} t$ collapse on top of a universal scaling function $f(x)$ (see Fig. 3). For selected values of $V_{0}$, $a / d$, and $n d^{3}$ [33] we determine $T_{c}$ also by calculating the 


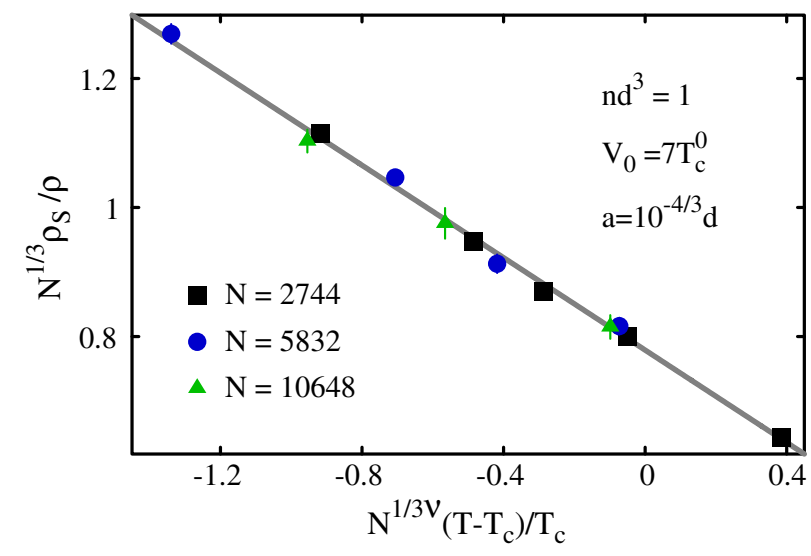

FIG. 3 (color online). Scaled superfluid fraction as a function of the scaled reduced temperature. Data obtained for different particle numbers $N$ collapse on top of the universal scaling function $f(x)$; see Eq. (2) (thick gray line).

fraction of particles with zero momentum $n_{0} / n$ (sometimes referred to as coherent fraction), which can be extracted from the long-distance behavior of the one-body density matrix $n_{1}\left(\mathbf{r}, \mathbf{r}^{\prime}\right)$ [22]. In the noninteracting case we obtain $T_{c}$ also by calculating via exact diagonalization the condensate fraction $n_{C} / n$, i.e., the fraction of particles in the Bloch state with zero quasimomentum [22,34]. All methods we employ to determine $T_{c}$ provide predictions which are consistent within statistical errors, typically of the order of $0.1 \%$.

In Fig. 4 we show the dependence of $T_{c}$ on the strength of the optical lattice potential $V_{0}$ at integer filling $n d^{3}=1$. Both in the interacting and in the noninteracting case $T_{c}$ monotonically decreases as $V_{0}$ increases. In moderately intense lattices as the one considered in this work, thermal

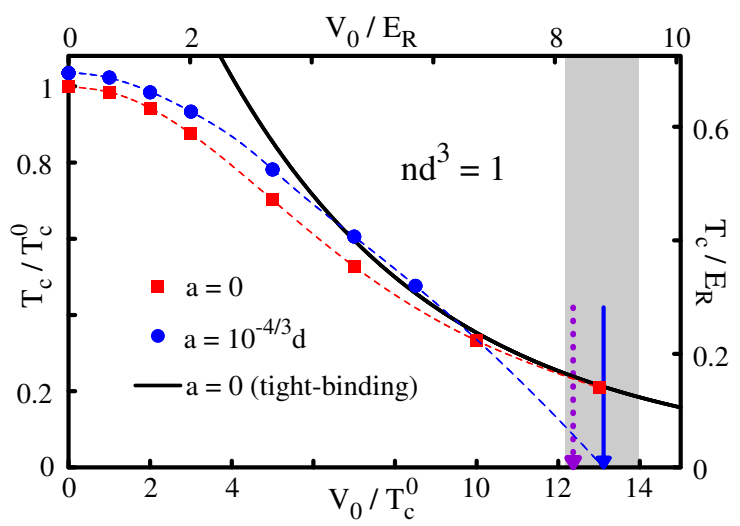

FIG. 4 (color online). Critical temperature as a function of the optical lattice intensity. The Mott insulator transition at $T=0$ is indicated by the solid blue vertical arrow (from Ref. [35]), by the dashed violet arrow (obtained via approximate mapping to the Bose-Hubbard model [18,36]) and by the shaded gray area (experimental result of Ref. [37] with error bar [38]). Thin dashed lines are guides to the eye. excitations populate higher Bloch bands making the single-band approximation invalid. Indeed we observe that the noninteracting critical temperature converges to the tight-binding result [18] only for $V_{0} \gtrsim 12 T_{c}^{0}$. Then it vanishes asymptotically in the large $V_{0}$ limit. In shallow optical lattices, $T_{c}$ is higher in the interacting case compared to the noninteracting case. As the lattice gets deeper $T_{c}$ rapidly decreases approaching zero at the quantum phase transition to the Mott insulator. In the proximity of the quantum critical point, finite-temperature PIMC simulations become impractical due to critical slowing down; even so the trend of our data at intermediate $T$ is consistent with the critical point predicted by previous Monte Carlo simulations of the ground state of the Hamiltonian (1) [35] and with the experimental result of Ref. [37]. The nonmonotonic dependence of $T_{c}$ as a function of the interaction parameter is highlighted in Fig. 1. Interaction effects are larger in an optical lattice than in the homogenous gas $\left(V_{0}=0\right)$. Indeed, if we assume a linear dependence $\Delta T_{c} / T_{c}^{0}=c n^{1 / 3} a=c a / d$ (here we consider the shift $\Delta T_{c}$ from the critical point at the given lattice depth $V_{0}$ and scattering length $a=0$ ), a best fit analysis in the range $0 \leq a / d \leq 0.01$ provides the coefficients $c=3.9(3)$ for $V_{0}=7 T_{c}^{0}$, and $c=1.24(7)$ for $V_{0}=0$ (see inset in Fig. 1). These results indicate a cooperative interplay between interactions and external potential. The superfluid density also shows a nonmonotonic dependence on $a / d$, even well below the critical temperature (see Fig. 5).

Figure 2 displays how $T_{c}$ varies with the lattice filling if the interaction strength is fixed at $a / d=10^{-4 / 3}$ and the optical lattice intensity at $V_{0}=7 T_{c}^{0}$. At low filling $\left(n d^{3} \approx 0.25\right)$ the critical temperature is almost unaffected by interactions. On the other hand, at high filling $\left(n d^{3} \approx 6\right)$ the role of interactions is dominant while the optical lattice becomes unimportant and $T_{c}$ approaches the transition of the homogeneous system. In the crossover region

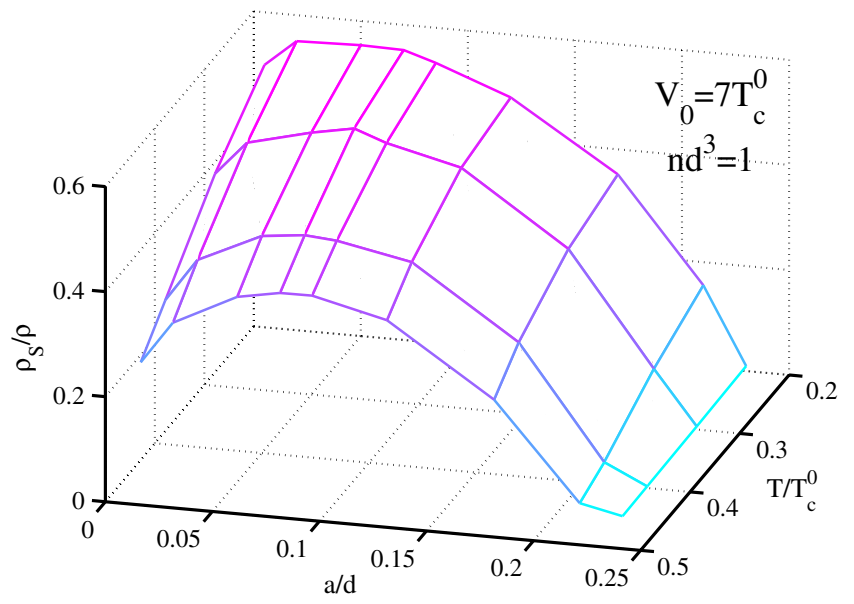

FIG. 5 (color online). Superfluid fraction as a function of the temperature $T / T_{c}^{0}$ and the interaction strength $a / d$. 
$0.5 \leq n d^{3} \leq 3$ the dependence of $T_{c}$ on the density is accurately described by a simple linear fitting function $T_{c}\left(n d^{3}\right)=E_{R}\left[0.376(2) n d^{3}+0.036(4)\right]$. It is worth noticing that in the optical lattice interactions can induce important changes of $T_{c}$, up to $40 \%$ at $n d^{3}=6$, much larger than in the homogeneous case. We explain this intriguing behavior of $T_{c}$ as a consequence of the screening of the external potential due to the interactions. This screening inhibits the suppression of $T_{c}$ which would otherwise be induced by the optical lattice if the particles were noninteracting.

Both the sharp positive shift of $T_{c}$ at fixed filling $n d^{3}=1$ and the linear dependence on $n d^{3}$ take place in a regime of small values of the diluteness parameter $n a^{3} \lesssim 5 \times 10^{-4}$. In this region universality in terms of the scattering length is preserved $[9,35]$ and the details of our model interparticle potential (e.g., the effective range and the scattering lengths in higher partial waves) are irrelevant. Hence, our results can be applied to describe experiments performed with ultracold atomic gases in the presence of broad Feshbach resonances.

In conclusion, we have investigated the combined effect of interactions and external periodic potentials on the superfluid transition in a 3D Bose gas. Previous approximate theoretical studies addressed the onset of superfluidity in weak unidirectional optical lattices [39], and the meanfield suppression of $T_{c}$ in combined harmonic plus opticallattice potentials [40]. The determination of $T_{c}$ in extended systems is a highly nonperturbative problem that can be rigorously solved only using unbiased quantum many-body techniques such as the PIMC method employed in this work. PIMC simulations have already been applied to investigate the superfluid transition in liquid ${ }^{4} \mathrm{He}$ [41], in dilute homogenous Bose gases $[8,42,43]$, in dipolar systems [44], and in disordered Bose gases [45,46]. So far, the theoretical studies and the experiments performed on optical lattice systems have been focused on the suppression of $T_{c}$ [47] and on the localization transition $[37,48]$, which take place in deep lattices and strong interatomic interaction. In this Letter, we show that correlations have a more intriguing effect on the quantum-coherence properties than what was previously assumed. In the regime of weak interactions, the superfluid fraction and the critical temperature are enhanced by interparticle repulsion. We attribute this phenomenon to the suppression of local density fluctuations. These fluctuations create large spatial regions with low density and are thus deleterious for establishing off-diagonal longrange order [42]. Instead, too strong interactions inhibit quantum pair exchanges and, hence, suppress superfluidity. In an optical lattice close to commensurability, the suppression of the local density fluctuations at weak interactions is even more pronounced that in a homogeneous system. We attribute to this effect the intriguing sharper upward shift of $T_{c}$ that we measure in optical lattices compared to the homogeneous case. We also find that, by tuning the filling factor above unity, one can reach a regime where the presence of the periodic potential becomes essentially irrelevant due to a screening effect caused by interactions. The recent realization of quasiuniform trapping potentials $[15,49]$ for atomic clouds and the development of new theories to describe the critical behavior in the presence of confinement [16] give us strong hope that these findings can be observed in experiments in the near future.

We acknowledge support by the Swiss National Science Foundation and by the ERC Advanced Grant SIMCOFE.

[1] S. Gandolfi, J. Carlson, and S. C. Pieper, Phys. Rev. Lett. 106, 012501 (2011)

[2] T. D. Lee and C. N. Yang, Phys. Rev. 105, 1119 (1957).

[3] J. O. Andersen, Rev. Mod. Phys. 76, 599 (2004).

[4] G. Baym, J.-P. Blaizot, M. Holzmann, F. Laloë, and D. Vautherin, Phys. Rev. Lett. 83, 1703 (1999).

[5] M. Holzmann, G. Baym, J.-P. Blaizot, and F. Laloë, Phys. Rev. Lett. 87, 120403 (2001).

[6] V. A. Kashurnikov, N. V. Prokof'ev, and B. V. Svistunov, Phys. Rev. Lett. 87, 120402 (2001).

[7] P. Arnold and G. Moore, Phys. Rev. Lett. 87, 120401 (2001).

[8] S. Pilati, S. Giorgini, and N. Prokof'ev, Phys. Rev. Lett. 100, 140405 (2008).

[9] S. Giorgini, J. Boronat, and J. Casulleras, Phys. Rev. A 60, 5129 (1999).

[10] S. Pilati, K. Sakkos, J. Boronat, J. Casulleras, and S. Giorgini, Phys. Rev. A 74, 043621 (2006).

[11] I. Bloch, J. Dalibard, and W. Zwerger, Rev. Mod. Phys. 80, 885 (2008).

[12] S. Giorgini, L. P. Pitaevskii, and S. Stringari, Phys. Rev. A 54, R4633 (1996).

[13] R. P. Smith, R. L. D. Campbell, N. Tammuz, and Z. Hadzibabic, Phys. Rev. Lett. 106, 250403 (2011).

[14] R. P. Smith, N. Tammuz, R. L. D. Campbell, M. Holzmann, and Z. Hadzibabic, Phys. Rev. Lett. 107, 190403 (2011).

[15] A. L. Gaunt, T. F. Schmidutz, I. Gotlibovych, R. P. Smith, and Z. Hadzibabic, Phys. Rev. Lett. 110, 200406 (2013).

[16] G. Ceccarelli, C. Torrero, and E. Vicari, Phys. Rev. B 87, 024513 (2013).

[17] In the Bose-Hubbard model $T_{c}$ decreases essentially monotonically, with possibly only a minor increase in the limit of weak interactions [18].

[18] B. Capogrosso-Sansone, N. V. Prokof'ev, and B. V. Svistunov, Phys. Rev. B 75, 134302 (2007).

[19] D. M. Ceperley, Rev. Mod. Phys. 67, 279 (1995).

[20] M. Boninsegni, N. V. Prokof'ev, and B. V. Svistunov, Phys. Rev. E 74, 036701 (2006).

[21] S. Pilati, S. Giorgini, M. Modugno, and N. Prokof'ev, New J. Phys. 12, 073003 (2010).

[22] See Supplemental Material at http://link.aps.org/supplemental/ 10.1103/PhysRevLett.112.170402 for details on the computational techniques.

[23] E. L. Pollock and D. M. Ceperley, Phys. Rev. B 36, 8343 (1987). 
[24] E. L. Pollock and K. J. Runge, Phys. Rev. B 46, 3535 (1992).

[25] B. D. Josephson, Phys. Lett. 21, 608 (1966).

[26] Large particle numbers $N \gg 100$ are required to remove the bias in the estimated $T_{c}$ due to the nonuniversal corrections to the scaling behavior, Eq. (2) $[8,27]$.

[27] S. Y. Bronin, B. V. Zelener, A. B. Klyarfeld, and V. S. Filinov, Europhys. Lett. 103, 60010 (2013).

[28] M. Campostrini, M. Hasenbusch, A. Pelissetto, P. Rossi, and E. Vicari, Phys. Rev. B 63, 214503 (2001).

[29] $T_{c}$ is not very sensible to the exact value of $\nu$, and we obtain compatible estimates if we fix $\nu=0.67 . f_{0}$ is related to the critical amplitude of the helicity modulus [30]. Our results do not compare well with the 3D $X Y$ model, possibly due to scaling corrections or to a more intricate critical behavior in the dilute Bose gas [31,32].

[30] M. E. Fisher, M. N. Barber, and D. Jasnow, Phys. Rev. A 8, 1111 (1973).

[31] P. B. Weichman, M. Rasolt, M. E. Fisher, and M. J. Stephen, Phys. Rev. B 33, 4632 (1986).

[32] K. K. Singh, Phys. Lett. A 193, 235 (1994).

[33] We consider $\left(V_{0} / T_{c}^{0}, a / d, n d^{3}\right)=\left(0,10^{-4 / 3}, 1\right),\left(7,10^{-4 / 3}, 1\right)$, $\left(7,10^{-4 / 3}, 6\right)$.

[34] I. Bloch, M. Greiner, and T. W. Hänsch, in Interactions in Ultracold Gases: From Atoms to Molecules, edited by M. Weidemüller and C. Zimmermann (Wiley-VCH, New York, 2003).

[35] S. Pilati and M. Troyer, Phys. Rev. Lett. 108, 155301 (2012).

[36] D. Jaksch, C. Bruder, J. I. Cirac, C. W. Gardiner, and P. Zoller, Phys. Rev. Lett. 81, 3108 (1998).
[37] M. J. Mark, E. Haller, K. Lauber, J. G. Danzl, A. J. Daley, and H.-C. Nägerl, Phys. Rev. Lett. 107, 175301 (2011).

[38] We obtained the value at $a / d=10^{-4 / 3}$ by interpolating six experimental data in the range $0.0053 \leq a / d \leq 0.053$. The width of the gray area is the error bar of the experimental point at $a / d=0.043$.

[39] O. Zobay and M. Rosenkranz, Phys. Rev. A 74, 053623 (2006).

[40] D. Baillie and P. B. Blakie, Phys. Rev. A 80, 031603(R) (2009).

[41] M. Boninsegni, N. Prokof'ev, and B. Svistunov, Phys. Rev. Lett. 96, 070601 (2006).

[42] P. Grüter, D. Ceperley, and F. Laloë, Phys. Rev. Lett. 79, 3549 (1997).

[43] K. Nho and D. P. Landau, Phys. Rev. A 70, 053614 (2004).

[44] A. Filinov, N. V. Prokof'ev, and M. Bonitz, Phys. Rev. Lett. 105, 070401 (2010).

[45] S. Pilati, S. Giorgini, and N. Prokof'ev, Phys. Rev. Lett. 102, 150402, (2009).

[46] G. Carleo, G. Boéris, M. Holzmann, and L. SanchezPalencia, Phys. Rev. Lett. 111, 050406 (2013).

[47] S. Trotzky, L. Pollet, F. Gerbier, U. Schnorrberger, I. Bloch, N. V. Prokof'ev, B. Svistunov and M. Troyer, Nat. Phys. 6, 998 (2010).

[48] M. Greiner, O. Mandel, T. Esslinger, T. W. Hänsch, and I. Bloch, Nature (London) 415, 39 (2002).

[49] I. Gotlibovych, T. F. Schmidutz, A. L. Gaunt, N. Navon, R. P. Smith, and Z. Hadzibabic, arXiv:1403.7081v1. 